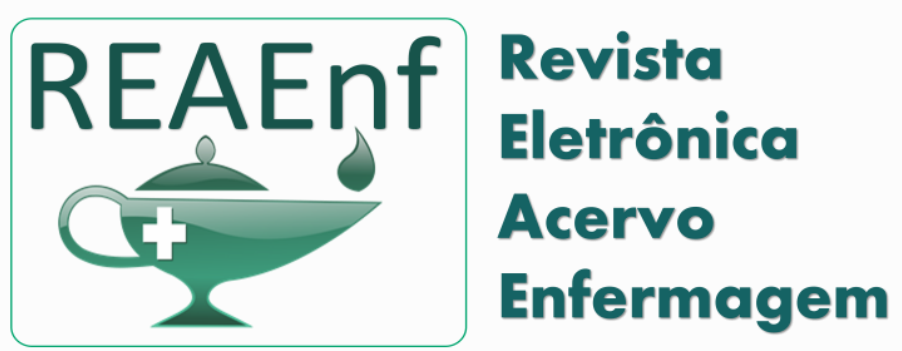

RELATO DE EXPERIÊNCIA

Recebido em: 10/2019

Aceito em: 10/2019

Publicado em: 11/2019

\title{
O PROCESSO DE IMPLANTAÇÃO DO AMBULATÓRIO DE FERIDAS DO CENTRO UNIVERSITÁRIO DO ESTADO PARÁ
}

The process of implantation of the ambulatory of wounds of the university center of the state of Pará El proceso de implantación del ambulatorio de heridas del centro universitario del estado de Pará

Haroldo Gonçalves de Jesus ${ }^{1 *}$, Augusto da Costa Pompeu ${ }^{1}$, Ivonete Vieira Pereira Peixoto $^{2}$, Margarete Carréra Bittencourt ${ }^{3}$.

\begin{abstract}
Resumo: Trata-se de um relato de experiência sobre a elaboração de protocolos para a implantação do ambulatório de Tratamento de Lesões na Pele como espaço para desenvolvimento profissional para discentes do Centro Universitário do Estado do Pará. Buscou-se destacar o processo de criação de protocolos para a implantação de um projeto assistencial-ambulatorial, educativo e de extensão no Centro de Especialidades Médicas do Centro Universitário do Estado do Pará. A construção do aparato gerencial e organizacional para ações dos profissionais enfermeiros, docentes e discentes do curso de enfermagem e sistematizou a assistência a ser prestada ao portador de feridas na instituição, fornecendo subsídios para a implementação deste serviço, dando diretrizes para o funcionamento, descrevendo a organização dos processos de trabalho tais como fluxo de atendimento e orientações técnicas; bem como indicando infraestrutura do espaço e equipamentos necessários para operacionalização do ambulatório. Logo a concepção deste relato se deve a ideia central de contribuir para discussões e reflexões sobre a importância do profissional enfermeiro para a saúde da população, através do desenvolvimento de protocolos e gestão de serviços.
\end{abstract}

Palavras-chave: Formulação de Projetos, Assistência Ambulatorial, Educação em Saúde.

\begin{abstract}
This is an experience report about the elaboration of protocols for the implantation of the Skin Injury Treatment Outpatient Clinic as a space for professional development for students from the University Center of the State of Pará. Protocols for the implementation of an outpatient, educational and extension project at the Center for Medical Specialties of the University Center of the State of Pará. The construction of the managerial and organizational apparatus for actions of nursing professionals, teachers and students of the nursing course and systematized the assistance to be provided to the patient with wounds in the institution, providing subsidies for the implementation of this service, giving guidelines for the operation, describing the organization of work processes such as care flow and technical guidance; as well as indicating infrastructure of the space and equipment needed for operation of the ambulatory. Thus, the conception of this report is due to the central idea of contributing to discussions and reflections on the importance of professional nurses for the health of the population, through the development of protocols and service management.
\end{abstract}

Keyword: Project Formulation, Ambulatory Care, Health Education.

\footnotetext{
${ }^{1}$ Centro Universitário do Estado do Pará (CESUPA), Belém-PA. *E-mail: haroldogonsalvez@gmail.com

2 Universidade Federal do Rio de Janeiro (UFRJ), Rio de Janeiro-RJ.

3 Universidade Federal do Pará (UFPA), Belém-PA.
} 
Resumen: Este es un informe de experiencia sobre la elaboración de protocolos para la implantación de la Clínica Ambulatoria de Tratamiento de Lesiones de la Piel como un espacio de desarrollo profesional para estudiantes del Centro Universitario del Estado de Pará. Protocolos para la implementación de un proyecto ambulatorio, educativo y de extensión en el Centro de Especialidades Médicas del Centro Universitario del Estado de Pará. La construcción del aparato gerencial y organizacional para acciones de profesionales de enfermería, docentes y estudiantes del curso de enfermería y sistematizado la asistencia que se brindará al paciente con heridas en la institución, brindando subsidios para la implementación de este servicio, brindando pautas para la operación, describiendo la organización de los procesos de trabajo, como el flujo de atención y la orientación técnica; además de indicar la infraestructura del espacio y el equipo necesarios para la operación del ambulatorio. Por lo tanto, la concepción de este informe se debe a la idea central de contribuir a las discusiones y reflexiones sobre la importancia de las enfermeras profesionales para la salud de la población, a través del desarrollo de protocolos y gestión de servicios.

Palabra clave: Formulación de Proyectos, Atención Ambulatoria, Educación Sanitaria.

\section{INTRODUÇÃO}

Atualmente as feridas configuram um problema crescente no Brasil, a incidência de portadores de doenças crônicas e degenerativas que ocasionam alterações na integridade da pele é alta, embora os registros de atendimento a estes usuários sejam escassos, é notório que este quadro constitui um sério problema de saúde pública. A busca por tratamentos relacionados a lesões de pele secundárias as patologias de base possui número elevado, e à medida que a ferida adquire cronicidade apresenta processo cicatricial prolongado, onerando maior gasto público. Além disso, tal condição interfere diretamente na qualidade de vida da população. Para evitar que isso ocorra, se faz necessário o envolvimento de uma equipe multiprofissional para propiciar uma assistência integral, atendendo as necessidades biopsicossociais, adquirindo caráter longitudinal para melhorar as condições de vida dos portadores de lesões crônicas (SANTOS AAR, et al., 2010).

A prevenção e o tratamento de feridas necessitam compor a rotina dos estabelecimentos de saúde, que para esse exercício devem configurar-se em ambientes que disponham de materiais e equipamentos adequados, que possuam uma equipe multidisciplinar com profissionais da saúde capacitados para esta finalidade, como é o caso de clínicas, unidades básicas de saúde da família, consultórios, sejam estes estabelecimentos de iniciativa pública ou privada. Neste espectro de oferta de serviços, a dermatologia na perspectiva da enfermagem, atualmente vem se desenvolvendo através da atuação da assistência direta do enfermeiro ao paciente em unidades ambulatoriais, domiciliares e hospitalares (CAVALCANTE BLL e LIMA UTS, 2012).

Nesse sentido, é conferido ao profissional enfermeiro membro da equipe multiprofissional atribuições assistenciais, procedendo com a avaliação e o tratamento de feridas, assim como orientação e supervisão da equipe de enfermagem e instrumentalização de cuidadores para a manutenção de curativos simples. Estas atividades comumente são realizadas em serviços de saúde de caráter ambulatorial, onde encontramse pré-estabelecidos protocolos que orientam a dinâmica de atenção ao portador de feridas (EVANGELISTA DG, et al., 2016).

Sturgess I e Proudfoot A (2009) citam as definições do Royal College of Physicians of London's, para conceituação da atenção ambulatorial, em livre tradução: "Atenção ambulatorial é o cuidado clínico que pode incluir o diagnóstico, observação, tratamento e reabilitação, não prevista no contexto da internação hospitalar tradicional ou serviços ambulatoriais tradicionais, e que podem ser prestados através da interface de atenção primária/secundária. "

Verifica-se em escala nacional que muitos dos pacientes portadores de feridas buscam a Atenção Primária como porta de entrada ou nela são acompanhados após atendimento de alta complexidade, o que confere a esse nível de atenção maiores envergadura e responsabilidade para assistência ao portador de lesões da pele (SANTOS ICRV, et al., 2014). 
Destaca-se que a nível regional, embora a Atenção Primária tenha avançado muito em garantir o acesso do cidadão às ações de atenção à saúde, o Estado do Pará carece ainda de serviços destinados a este grupo de pacientes, bem como informações sistematizadas sobre a caracterização da população atendida, além da carência de recursos disponíveis para prestação dessa assistência.

Devido à compreensão da necessidade de serviços ambulatoriais que ofertem de forma equânime e integral a assistência a pacientes portadores de lesões crônicas além da formação de recursos humanos para atender às necessidades de saúde da população, surgiu o interesse em elaborar o regulamento de normas e rotinas e protocolo assistencial para a implantação do ambulatório de atenção interdisciplinar e interprofissional, envolvendo estratégias de educação permanente, proporcionando atendimento de interconsultas de profissionais de saúde do Centro de Especialidades Médicas do Centro Universitário do Pará (CESUPA), orientando-os e capacitando-os nas condutas a serem executadas nos pacientes portadores de feridas, em especial, feridas crônicas além do enriquecimento curricular. Garantindo a formação profissional alinhada a qualidade da assistência prestada ao portador de ferida relacionando diretamente à capacitação/qualificação do profissional enfermeiro.

\section{RELATO DE EXPERIÊNCIA}

Trata-se de um relato de experiência, de abordagem qualitativa que descreve aspectos vivenciados pelos graduandos de enfermagem do CESUPA, destacando o processo de criação de protocolos para a implantação de um projeto assistencial-ambulatorial, educativo e de extensão no Centro de Especialidades Médicas do CESUPA (CEMEC).

O ponto de partida para implantação do ambulatório de feridas crônicas ocorreu com a solicitação de contato inicial à coordenação do CEMEC e a Pró-reitoria do CESUPA, através de uma carta encaminhada pelos graduandos via protocolo interno da instituição, apresentando as contribuições que a implantação do projeto traria para a instituição e comunidade acadêmica.

Posteriormente, a próxima etapa consistiu em reuniões e visitas ao CEMEC, para investigar a abordagem que a equipe do estabelecimento aplicava ao deparar-se com clientes portadores de lesões de pele. Verificou-se que havia um elevado número de clientes idosos com patologias de base incluindo doença vascular, insuficiência venosa, hipertensão arterial sistêmica e diabetes, que realizavam acompanhamento regular no CEMEC para controle das condições crônicas e quando estes apresentavam alterações de pele, eram realizados atendimentos pontuais sem a existência de um fluxo ordenado para o acompanhamento do processo cicatricial.

Concomitante as reuniões, realizou-se embasamento teórico fundamentado na literatura disponível em fontes de textos bibliográficos procurados nas bases de dados Scielo, Bireme, periódicos da Capes, revistas eletrônicas, artigos científicos. Os conhecimentos adquiridos ajudaram a identificar, agrupar e sistematizar informações existentes sobre o assunto, para que fosse elaborada a proposta de regulamento para a implantação do ambulatório de atenção ao paciente portador de ferida na instituição bem como o protocolo assistência.

A construção do regulamento para o funcionamento do ambulatório tornou-se a ferramenta gerencial tendo em vista que dispõe sobre a identificação do serviço e sua finalidade, a organização e composição da equipe multiprofissional, bem como delineia as atribuições e competências da equipe. Desta maneira também foi estabelecido no regulamento um fluxograma de atendimentos, que garante a dinâmica de atendimento e acompanhamento do processo cicatricial orientando as condutas tomadas pela equipe. Esta estratégia corrobora para inserção do corpo docente e discente de enfermagem da instituição, operacionalizando o serviço de forma permanente e garantindo a qualificação do profissional no contexto da formação acadêmica, pois na prática diária, o cuidar de feridas, nos diferentes níveis dos serviços de saúde, é de responsabilidade do enfermeiro e requer deste profissional qualificações adequadas. 
O enfermeiro tem papel fundamental na atuação preventiva, avaliação e tratamento de feridas, exigindo deste profissional conhecimentos técnico-científicos. Para realizar o melhor cuidado a cada paciente surge a necessidade de protocolos de avaliação e tratamento, que orientem o exercício profissional e qualifique a assistência. A criação do protocolo utilizado, foi guiada pelas demandas encontradas no estabelecimento, norteado pelos princípios e diretrizes do Sistema Único de Saúde e embasados em protocolos de serviços já consolidados, como o Protocolo de cuidados de feridas da Secretaria Municipal de Saúde de Florianópolis (2007).

Além de permitir a efetivação da oferta de um serviço pioneiro no estado do Pará, a implantação do ambulatório consolida o aprendizado aliando teoria e prática proporcionando aos seus discentes a interação com outras áreas do conhecimento, estimulando a prática do diálogo interdisciplinar. O serviço também favorece a abertura de um amplo campo de pesquisa, ao reunir informações sobre os atendimentos de portadores de feridas gerando futuramente estatísticas acerca da temática.

\section{DISCUSSÃO}

Discorrer sobre o tratamento de feridas é tarefa árdua, pois a temática possui especificidades que requerem dos profissionais um conhecimento amplo e profundo quanto a anatomia, a fisiologia e as diferentes mutações desencadeadas no tecido epitelial, induzidas por agentes e fatores externos e internos. Além de apresentar peculiaridades e alterações em decorrência da região que reveste, da idade do indivíduo portador da lesão crônica, ou de algumas situações e estados orgânicos.

Ao tratarmos diretamente da assistência de enfermagem a pacientes portadores, o profissional necessita planejar e qualificar suas ações. Uma vez que a assistência ao portador de lesões de pele, assim como as diversas formas de tratamento, exige do enfermeiro um conjunto de estratégias que permitam alcançar os objetivos propostos, por meio da sistematização da assistência de enfermagem (SILVA ES, 2011).

Para o alcance da assistência sistematizada ressalta-se a importância da aplicação do processo de enfermagem no atendimento aos pacientes, garantindo a abordagem integral mediante a identificação das necessidades apresentadas e a qualidade do cuidado. Através de atitudes, métodos e técnicas de prevenção, proteção, promoção e condutas de reabilitação, o enfermeiro busca o equilíbrio de uma condição saudável ao ser humano, por meio de planejamento, organização e execução de seu trabalho, embasando-o na problematização advinda das relações saúde e doença.

Os profissionais precisam dispor de conhecimento e de recursos materiais para que possam prestar uma assistência com abordagem integral do indivíduo, garantindo a continuidade da atenção. Neste sentido, entendemos que a atualização constante de conhecimentos, bem como a implantação e implementação de protocolos e normatizações se fazem necessários (ZUFFI FB, 2009).

Na perspectiva de realizar o melhor cuidado para cada paciente, surge à necessidade de protocolos de avaliação e tratamentos de feridas para unificar e contribuir para um atendimento mais prático e qualificado. A criação do protocolo guiada por objetivos ligados a equipe multiprofissional, da instituição garante a qualidade do atendimento a ser prestado no ambulatório.

O protocolo é um plano exato e detalhado para o estudo de um problema de saúde, cujo o objetivo é a implementação de um esquema terapêutico, que maximiza o potencial humano e reduz custos, resultando na sistematização da assistência, uma vez que requer o registro dos achados clínicos e das informações coletadas na anamnese (BORGES EL, et al., 2008).

A construção destes instrumentos, fomenta a funcionalidade do serviço, implementando diretrizes para o funcionamento do serviço, descrevendo a organização dos processos de trabalho tais como fluxo de atendimento e orientações técnicas; bem como indicando infraestrutura do espaço e equipamentos necessários, além de recursos impressos e insumos gerais para operacionalização do ambulatório.

Constituindo uma valiosa ferramenta de educação permanente e serve de guia para adoção da melhor conduta, preservando a autonomia do profissional de saúde. Tampouco, pode-se esquecer de que o seu 
processo de construção possibilita vasta aprendizagem organizacional, na medida em que prescreve os melhores recursos a serem utilizados, e que garante maior probabilidade de se alcançar os resultados pretendidos.

A criação dos protocolos para tratamento e acompanhamento de feridas é de extrema necessidade ao serviço e ao corpo docente e discente de enfermagem da instituição, uma vez que estes estarão inseridos no serviço, que ao alinhar suas ações ao protocolo configura um ambiente seguro para o acompanhamento e controle dos casos. Além disso, este instrumento colabora com o processo de trabalho cotidiano da equipe multiprofissional, garantindo atendimento aprimorado à população (MORAIS GFC, et al., 2008).

Assim os docentes e discentes da instituição podem desenvolver um trabalho de excelência e com resultados positivos. Tendo em vista que o protocolo pré-estabelece quem avaliará a ferida e indicará o tratamento, bem como caracterizará a clientela, permitindo a padronização dos materiais específicos para prevenção e tratamento de feridas, levando em conta a relação custo-benefício. Significa dizer que o protocolo se torna a ferramenta que garante a sistematização da assistência, ao estabelecer também a documentação das informações colhidas e as condutas a serem adotadas no decorrer do atendimento e do tratamento.

Portanto a elaboração do aparato gerencial e organizacional para ações dos profissionais enfermeiros, docentes e discentes do curso de enfermagem e sistematização da assistência a ser prestada ao portador de feridas no CEMEC, fornece os subsídios necessários a implantação deste serviço ambulatorial pelas instâncias superiores da instituição CESUPA, onde os portadores de lesões agudas e crônicas serão avaliados por alunos do curso de enfermagem e juntamente com a equipe de docentes interdisciplinares: nutrição, enfermagem e fisioterapia.

\section{CONSIDERAÇÕES FINAIS}

A concepção deste relato de experiência se deve a ideia central de que ele possa contribuir para discussões e reflexões sobre a importância do profissional enfermeiro para a saúde da população e, principalmente, para os portadores de lesões tegumentares, através do desenvolvimento de protocolos e gestão de serviço, testemunhando a evolução da profissão através da assistência no consultório, superando o pensamento do tratamento de lesões tegumentares como uma prática enclausurada no âmbito hospitalar, trazendo a proposta de aperfeiçoar os cuidados prestados garantindo maior qualidade para a assistência de enfermagem. A implantação de protocolos é uma decisão estratégica de fortalecimento das melhores práticas assistenciais e essa iniciativa é liderada pelo enfermeiro. Representa um esforço institucional que integra várias equipes profissionais e a implantação e adesão de protocolos nas unidades de saúde em diversos níveis deve contar com o apoio do gestor e da instituição que representa. Considera-se que a construção destes protocolos assistenciais de feridas é muito significativa para nortear a conduta dos profissionais de enfermagem, ressaltando que toda a equipe trabalha em conjunto guiada cientificamente, com o objetivo de tratar a lesão adotando uma visão holística da pessoa.

\section{REFERÊNCIAS}

1. ABREU AM, et al. Atendimento a pacientes com feridas crônicas nas salas de curativos das policlínicas de saúde. Revista Brasileira de Pesquisa em Saúde, v. 152, p. 42-49, jun. 2013.

2. BORGES EL, et al. Feridas: como tratar. 2. Ed. Belo Horizonte: Coopmed, 2008.

3. CAVALCANTE BLL, LIMA UTS. Relato de experiência de uma estudante de enfermagem em um consultório especializado em tratamento de feridas. J Nurs Health, Pelotas (RS) 2012 jan/jun;1(2):94103.

4. DUTTON M, et al. The role of the wound care nurse: an integrative review. Br J Community Nurs 2014; Suppl: S39-40, S42-7.

5. EVANGELISTA DG, et al. Impacto das feridas crônicas na qualidade de vida de usuários da Estratégia de Saúde da Família. Rev Enferm Cent Oest Min. 2012; 2(2):254-63. 
6. KOBAYASHI RM, LEITE MMJ. Desenvolvendo competências profissionais dos enfermeiros em serviço. Rev. Bras. Enferm. 2010; 63: 243-9.

7. MORAIS GFC, et al. Avaliação de feridas pelos enfermeiros de instituições hospitalares da rede pública. Texto Contexto Enfermagem, Florianópolis, v. 17, n. 1, mar. 2008.

8. SANTOS AAR, et al. Avaliação e tratamento de feridas: o conhecimento de acadêmicos de enfermagem. Rev enferm UERJ 2010; 18: 547-52.

9. SANTOS ICRV, et al. Caracterização do atendimento de pacientes com feridas na atenção primária. Rev Rene. 2014 jul-ago; 15(4):613-20.

10. SILVA ES. Feridas crônicas: conhecimento e importância do tratamento sob a ótica do paciente. 2011, Monografia (Especialização em Saúde Pública) - Programa de Pós-Graduação em Saúde, Faculdade Adventista da Bahia, Cachoeira, 2011; 63 p.

11. STURGESS I, PROUDFOOT A. Ambulatory care: What is it and do we need it? Br. J. Hosp. Med. (Lond). 2009 Jan;70: S15-8.

12. TUYAMA LY, et al. Feridas crônicas de membros inferiores: proposta de sistematização de assistência de enfermagem a nível ambulatorial. Nursing: rev. Técnico científica enferm. 2004 Ago; 75 (7): 46-50.

13. ZUFFI FB. A atenção dispensada aos usuários com úlcera venosa: percepção dos usuários cadastrados nas equipes de saúde da família. Dissertação (mestrado em enfermagem) - Escola de Enfermagem de Ribeirão Preto; Universidade de São Paulo, Ribeirão Preto (SP), 2009, 129 f. 